\title{
ESTIMATION OF VERTICAL DEFLECTIONS IN CONCRETE BEAMS THROUGH DIGITAL CLOSE RANGE PHOTOGRAMMETRY
}

\author{
I. Detchev ${ }^{\mathrm{a}, *}$, A. Habib ${ }^{\mathrm{a}}$, M. El-Badry ${ }^{\mathrm{b}}$ \\ ${ }^{a}$ Department of Geomatics Engineering \\ ${ }^{\mathrm{b}}$ Department of Civil Engineering \\ University of Calgary, 2500 University Drive NW, \\ Calgary, Alberta, T2N 1N4 Canada - (i.detchev, ahabib)@ucalgary.ca
}

Commission V, WG V/3

KEY WORDS: camera, laser scanning, convergent, acquisition, monitoring, surface, reconstruction, point cloud

\begin{abstract}
:
Deformation monitoring, and in general structural health monitoring, of civil infrastructure systems is important in terms of both safety and serviceability. Traditionally, large structures have been monitored using surveying techniques, while fine-scale monitoring of structural components has been done with geotechnical instrumentation. This paper reviews the advantages and disadvantages of using remote sensing methods, such as terrestrial laser scanning and digital close range photogrammetry, for the purposes of precise $3 \mathrm{D}$ reconstruction and the estimation of deflections in structural materials. It is also shown how a low-cost setup of multiple digital cameras and projectors can be used for the monitoring of concrete beams subjected to different loading conditions by a hydraulic actuator. The photogrammetric system used does not require any physical targets other than for the purposes of establishing the relative orientation between the involved cameras. The setup was tested in two experiments, and the beam deflections resulted from the photogrammetric system were compared to the ones from a set of one-dimensional laser transducers and a terrestrial laser scanner. The experiments proved that it was possible to detect sub-millimetre level deformations given the used equipment and the geometry of the setup.
\end{abstract}

\section{INTRODUCTION}

Health monitoring of infrastructure systems is an important task and is usually done for two reasons. The first one is safety (i.e. testing structural components or down-scaled models of designed structures in order to estimate their maximum loading capacity), and the second one is serviceability (i.e. performing regularly scheduled monitoring procedures in order to assess whether any maintenance is required on an already built structure) (Park et al., 2007).

Traditionally, large structures such as dams, bridges, open-pit mines or high-rise buildings have been monitored for overall deformations through ground based surveying techniques, i.e. measurement of horizontal angles (or directions), zenith angles, slope distances and height differences using precision grade total stations or theodolites and precision levels (Ebeling et al., 2011; González-Auilera et al., 2008). Recently, these techniques have been complemented by the use of global positioning methods, where geodetic grade receivers and antennas collect signals from all visible satellites in a static mode over long periods of time (Bond et al., 2008; Ebeling et al., 2009; Kim et al., 2003). Despite the wide variety of available surveying instruments and the well-established data processing and network adjustment techniques, they can only observe a limited number of points, which need to be carefully selected at the specific areas of anticipated deformation.

On the fine-scale side of structural health monitoring, the appearance of cracks and the failure of foundations, walls, support columns or structural components in general, have been

\footnotetext{
* Corresponding author.
}

measured via geotechnical techniques, for example using tiltmeters, micrometers, inclinometers, wire strain gauges or extensometers (González-Auilera et al., 2008; Maas and Hampel, 2006). Lately, optical fibre sensors (Inaudi et al., 1998; Shen et al., 2010) and inductive laser transducers or linear voltage displacement transducers (LVDT) have also been implemented (Mills et al., 2001). These processes usually have either low or no redundancy, they can measure changes only at the point, where they are installed, and typically the measurement is done in only one direction (Gordon and Lichti, 2007; Maas and Hampel, 2006). Moreover, due to the very close proximity of the used instrumentation to the monitored objects of interest, there is a high risk of it being damaged or destroyed by any failing components (Gordon and Lichti, 2007).

In order to avoid the above mentioned problems in largestructure and in fine-scale deformation monitoring, remote sensing techniques can be used. In the last decade or so, sensors in the realm of digital photogrammetry and laser scanning have started to be integrated into structural health monitoring systems. The potential advantages of such remote sensing methods using cameras or laser scanners, are that the object of interest does not have to be accessed while being measured, and that permanent visual records (either images or point cloud scenes) of it are established for each observed epoch of time. Also, objects can be reconstructed and deformations can be detected in 3D with a great amount of redundancy, and the overall precision can be evaluated through a least squares adjustment. 


\section{PREVIOUS RESEARCH}

Mills et al. (2001) used a single small format digital camera attached to a moving crane in order to map a test bed in a pavement rolling facility. Given the used geometry, the experiment resembled near vertical airborne mapping, and due to the small sensor size used at the time the height-base ratio was undesirable, so the overall reconstruction root mean square error (RMSE) for the performed experiments was about 2-3 $\mathrm{mm}$. Fraser and Riedel (2000) performed near real-time multiepoch deformation monitoring of heated steel beams while cooling off in a thermal test facility. Three digital cameras positioned at convergent geometry, and specially designed targets for such high temperature environment were used to obtain a final precision for the reconstructed object space coordinates of $1 \mathrm{~mm}$. Jáuregui et al. (2003) used double sided targets and measured deflections in steel beams at an RMSE of $0.5-1.3 \mathrm{~mm}$ in an indoor laboratory. In addition, they also managed to measure the deflections in bridge girders on a highway at an RMSE of 0.5-1.5 mm.

Despite the low-cost, the quick data acquisition, and the high accuracy of $3 \mathrm{D}$ reconstruction, traditionally signalized targets are necessary in digital photogrammetry. Another downside is that the scale in a photogrammetric bundle adjustment must be defined through the use of an additional instrument (GonzálezAuilera et al., 2008) (e.g. total station or a steel chain) or a specially designed invar bar must be observed in the acquired images (Gordon and Lichti, 2007). Also, since cameras are passive sensors, deformation monitoring using photogrammetry cannot be accomplished outdoors at night (Park et al., 2007) or in a dark laboratory facility. This is why some researchers have lately preferred the use of terrestrial laser scanning for deformation monitoring purposes. Laser scanning is independent of natural light (Park et al., 2007), it is entirely non-contact and, in most cases, it is also a targetless method of directly measuring $3 \mathrm{D}$ coordinates with full surface coverage at a very high frequency (Gordon and Lichti, 2007). Its single point positioning has poor quality (Gordon and Lichti, 2007; Monserrat and Crosetto, 2008; Park et al., 2007), but this could be overcome by performing geometrical fitting of the collected data. For example, Gordon and Lichti (2007) used the fundamental beam-deflection equations and implemented least squares curve fitting to increase the deflection precision of observed timber and concrete beams by up 20 fold, resulting in RMSE of 2-3 $\mathrm{mm}$ or $0.3 \mathrm{~mm}$ depending on the quality of the laser scanner used. Park et al. (2007) fitted planes to the side and the top of steel beams, and intersected them to produce lines in 3D. The accuracy of this method was under $1 \mathrm{~mm}$ at a scanning distance of several metres. Laser scanners however have limited range and sometimes multiple point clouds must be collected in order to cover the area of interest. This requires a co-registration procedure. At the same time, the produced point clouds are irregular and there is no point-to-point correspondence between overlapping scenes or scenes from different measuring epochs. This is why algorithms such as the iterative closest point (ICP) (Besl and McKay, 1992; Chen and Medioni, 1992) must be used to register the point clouds to a single reference frame. Monserrat and Crosetto (2008) used this idea to co-register point clouds from different epochs, and estimated the deformation parameters using local least squares surface matching (Gruen and Akca, 2005) with precision of 1-2 $\mathrm{cm}$ over scanning distances of 100-200 m.
The biggest disadvantage of laser scanning instruments, however, remains their very high cost. This is why facilities, which cannot afford to purchase one, must stick to the digital photogrammetric means of $3 \mathrm{D}$ reconstruction. In order to achieve targetless point cloud generation, structured light can be used to supply surfaces with artificial texture sufficient for subsequent image matching by the means of projecting a random pattern during the data collection (Lin et al., 2008). In this way, Lin et al. (2008) monitored the deformations of membrane roofs with a precision of 1.3-1.6 mm, where the scale was defined by imaging the footprint of a reflectorless total station. As stated in their article, the precision could have been significantly improved if more cameras were available. The present study is thus motivated to investigate the potential for deformation monitoring of statically loaded concrete beams using a close range photogrammetric system consisting of multiple inexpensive off-the-shelf digital cameras and projectors.

\section{PHOTOGRAMMETRIC SYSTEM SETUP}

A multiple camera and projector photogrammetric system had to be installed on both sides of a $250 \mathrm{kN}$ hydraulic actuator with an attached spreader beam (see Figure 1) in a structures laboratory. It was to be used in photographing concrete beams (see example in Figure 2) under different loading conditions, where the changing loads would be applied by the abovementioned actuator. A metal frame had to be designed and welded around the actuator (see Figure 3) in order to hoist the cameras and the projectors in secure positions above the beam being tested. Observing the top surface of the beam was preferred to observing its longitudinal side, because the bulk of the deformation was naturally anticipated to be along the gravity vector.

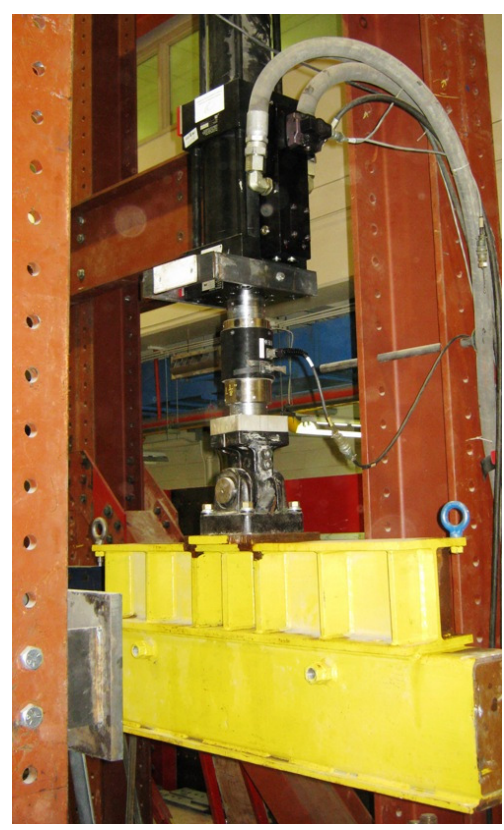

Figure 1. Spreader beam (in yellow) attached to a hydraulic actuator (in black and silver) 


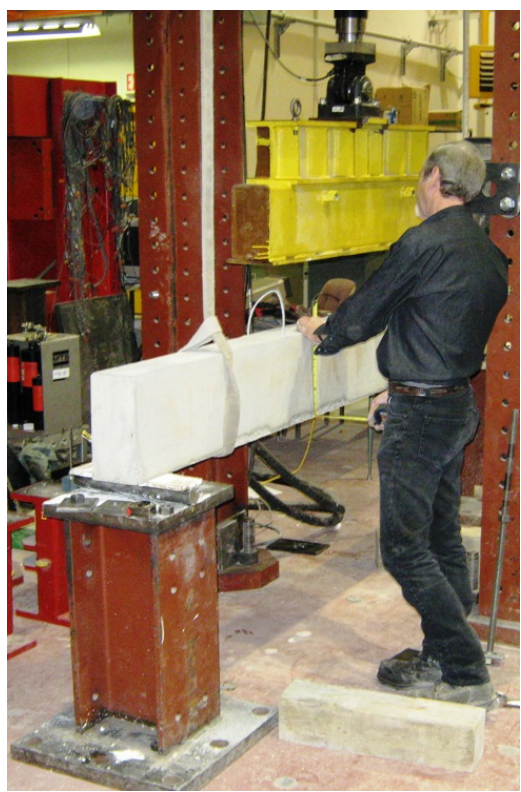

Figure 2. Placement of a concrete beam to be used for the experiment

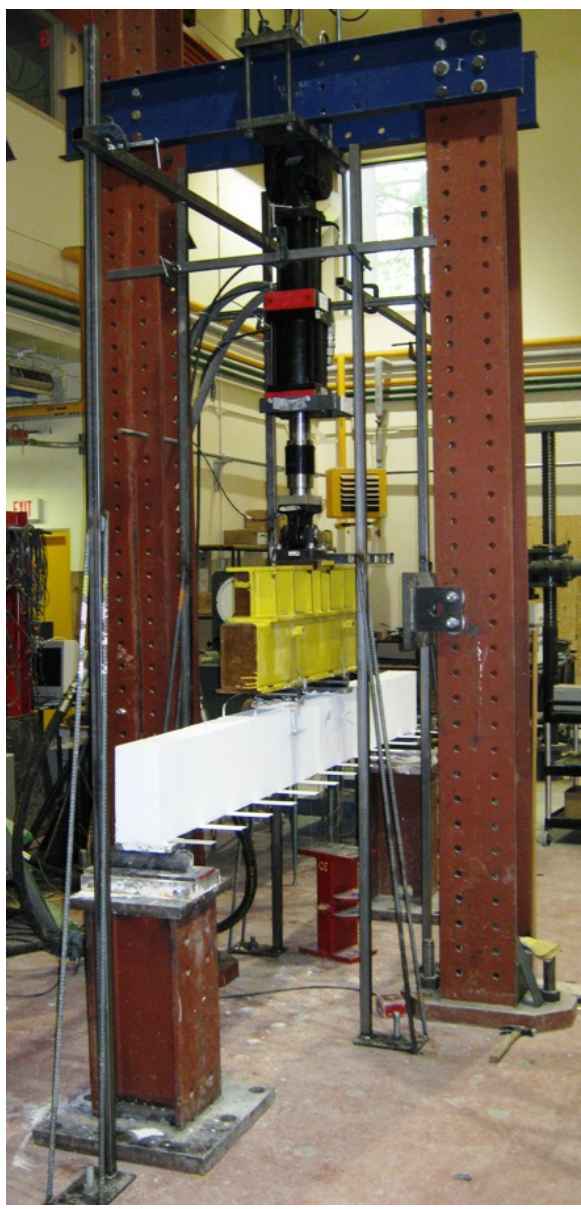

Figure 3. Part of the metal frame built to hoist the cameras and the projectors of the digital photogrammetric system
Four digital cameras were mounted on tripod heads, and attached to the west part of the metal frame (Figure 4), while three other cameras were mounted and attached to the east part. One digital projector was also attached on each side of the supporting frame.

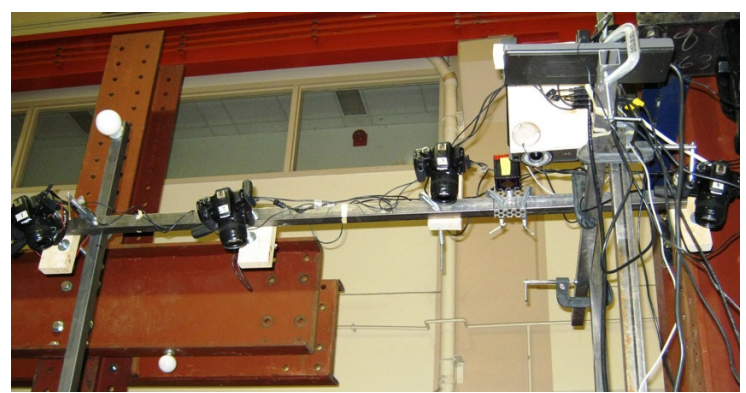

Figure 4. Example of the multiple camera and projector setup on the west part of the metal frame

Before the cameras were used for this project, they were geometrically calibrated. It was also verified that their internal orientation parameters (IOPs) were stable, i.e. they did not change significantly over time. After the cameras were installed on the metal frame, they had to have their relative orientation estimated. The location and the orientation, i.e. the exterior orientation parameters (EOPs), of each camera were obtained through a bundle block adjustment procedure using checkerboard paper targets. These signalized targets were spread out on the lab floor, on the concrete beam and on the spreader beam attached to the hydraulic actuator (see Figure 5). The scale for the bundle block adjustment was defined by several distances measured with a steel tape between some of the targets on the floor. Since the cameras were rigidly mounted on the metal frame, their relative EOPs were assumed not to change for the full duration of each experiment. This is why the bundle block adjustment was done only once before the beginning of each of the conducted experiments, and the paper targets were removed right before the commencement of the actual beam testing (see Figure 6), i.e. they were not used for reconstruction purposes.

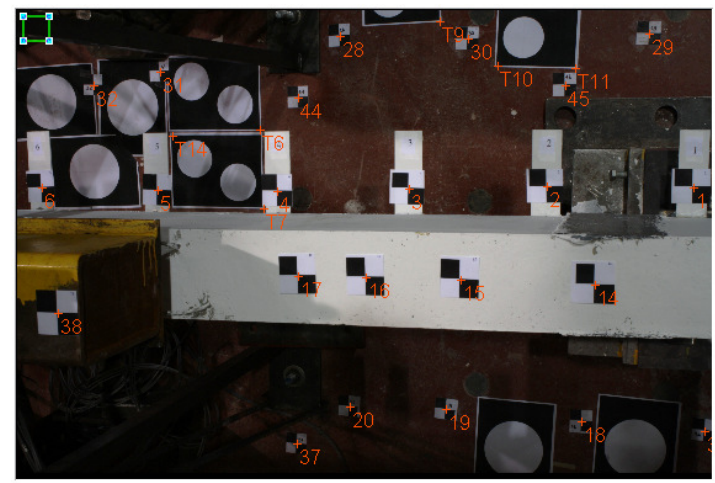

Figure 5. Example of the distribution of signalized targets on the floor, on the concrete beam, and on the spreader beam 


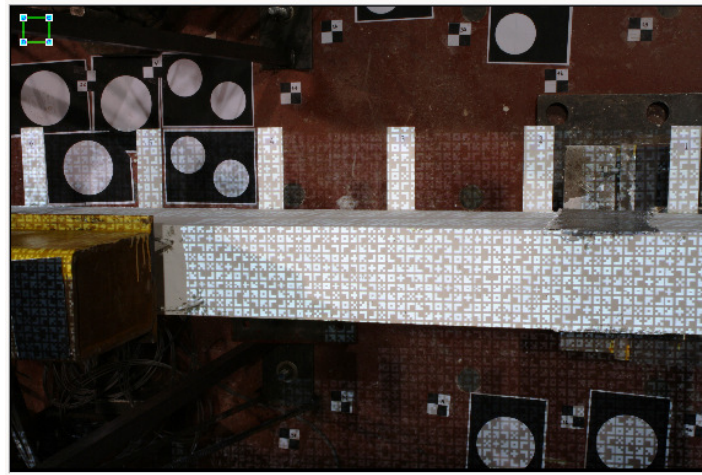

Figure 6. Example of the absence on signalized targets and the presence of a projected random pattern on the surface of the concrete beam during the testing

As mentioned in the previous section, the digital projectors were used to project a random pattern image in order to facilitate the matching problem of conjugate features between the overlapping camera images. As seen from Figure 6, the projected pattern image added artificial texture to the otherwise white-washed concrete surface, and this made the subsequent matching portion of the data processing not only possible, but also reliable.

\section{EXPERIMENTAL RESULTS}

There were two beam deformation monitoring experiments conducted. One of them used a concrete beam and the other one used a concrete beam with a polymer sheet glued to its underside. Both beams were-white washed, three metres long (with cross sections of $30 \mathrm{~cm} \times 15 \mathrm{~cm}$ ), and were statically loaded by the hydraulic actuator. Given the hydraulic actuator setup at hand, the spreader beam attached to it was obstructing a large portion of the top surface of the placed concrete beams. This is why, in addition to observing the visible portions of the beam surface, the cameras had to also observe $5 \mathrm{~cm} \times 15 \mathrm{~cm}$ white-washed aluminium plates attached at $25 \mathrm{~cm}$ intervals to the bottom surface of the beams. These metal plates served effectively as offset witnesses to the bottom surfaces of the real beams.

The two experiments were divided into three phases:

- Phase I - settling the beam on the given support by loading it from $0 \mathrm{~mm}$ stroke up to $3 \mathrm{~mm}$ stroke and releasing it back to $0 \mathrm{~mm}$ stroke two consecutive times;

- $\quad$ Phase II - loading the beam up to failure at regular intervals of $5 \mathrm{~mm}$ strokes, and

- $\quad$ Phase III - releasing the beam down to $0 \mathrm{~mm}$ stroke (i.e. until the permanently damaged beam separated from the spreader beam attached to the hydraulic actuator).

For each of the loading epochs, there were several sensors external to the beams that were used to collect data in order to measure the beam deflections:

- Three 1D laser transducers (positioned under plates $\# 5$, \#7, and \#9);

- Seven digital cameras, synchronized to operate simultaneously, and two digital projectors;

- Two terrestrial laser scanners, and
- Two range cameras.

The processing required for the photogrammetric reconstruction involved corner detection in all images, images matching between all image pairs, corner tracking for the detected corners common to at least three consecutive images, and multiple light ray intersection for computing the $\mathrm{X}, \mathrm{Y}$, and $\mathrm{Z}$ coordinates of the unknown object points using the known IOPs and EOPs for each camera. The above-mentioned processing steps were automatic, except for the selection of the region of interest in the images, which was done manually. Examples of the resulted 3D reconstruction can be seen in Figure 7 and Figure 8. Note that plate \#7, i.e. the centre plate, was not reconstructed, because it was not observed by three neighbouring cameras.

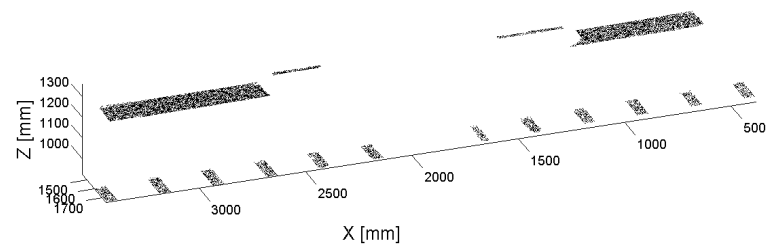

Figure 7. Example of the photogrammetric reconstruction for the $0 \mathrm{~mm}$ load stroke epoch for one of the experiments

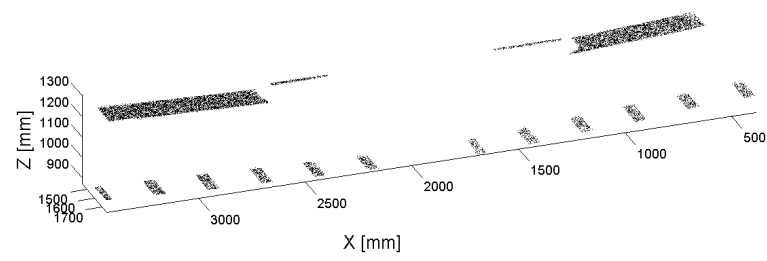

Figure 8. Example of the photogrammetric reconstruction for the $65 \mathrm{~mm}$ load stroke epoch for one of the experiments

The different reconstructed surfaces were first segmented, and then the $\mathrm{X}, \mathrm{Y}$, and $\mathrm{Z}$ object coordinates of all the points belonging to the same plates were averaged thus computing the centroids of each of the available plates. Subtracting the $Z$ value of the centroids of each plate for the first epoch from the $\mathrm{Z}$ values of the centroids for the corresponding plates for the rest of the epochs yielded the beam deflections $(\delta \mathrm{Z})$ at each available plate for each observed epoch. Example plots (produced using the cubic spline function in MATLAB) of Phase II for the two experiments can be seen in Figure 9 and Figure 10.

In order to check the quality of the photogrammetric reconstruction, the $\delta Z$ values for plates \#5 and \#9 resulted from the photogrammetric system were compared to the $\delta \mathrm{Z}$ values computed from the 1D laser transducers (see Table 1). For the first experiment, the mean for the difference values ranged between $-0.06 \mathrm{~mm}$ and $-0.10 \mathrm{~mm}$, with standard deviations of $\pm 0.11 \mathrm{~mm}$ and $\pm 0.08 \mathrm{~mm}$, for overall RMSE values of $0.12 \mathrm{~mm}$ and $0.13 \mathrm{~mm}$. The results from the second experiment were not as good - the mean for the difference values ranged between $0.9 \mathrm{~mm}$ and $1.9 \mathrm{~mm}$, with standard deviations of $\pm 0.6 \mathrm{~mm}$ and $\pm 1.1 \mathrm{~mm}$, for overall RMSE values of $1.1 \mathrm{~mm}$ and $2.5 \mathrm{~mm}$. The reason for the higher discrepancies for the second experiment was that after the $25 \mathrm{~mm}$ load stroke, the polymer sheet separated from the concrete beam, and the metal plates were 
tilted, so their centroids were no longer valid offset witnesses to the bottom surface of the beam. Hence, the deflections computed from the centroids of the metal plates no longer represented the deflections measured by the 1D laser transducers.

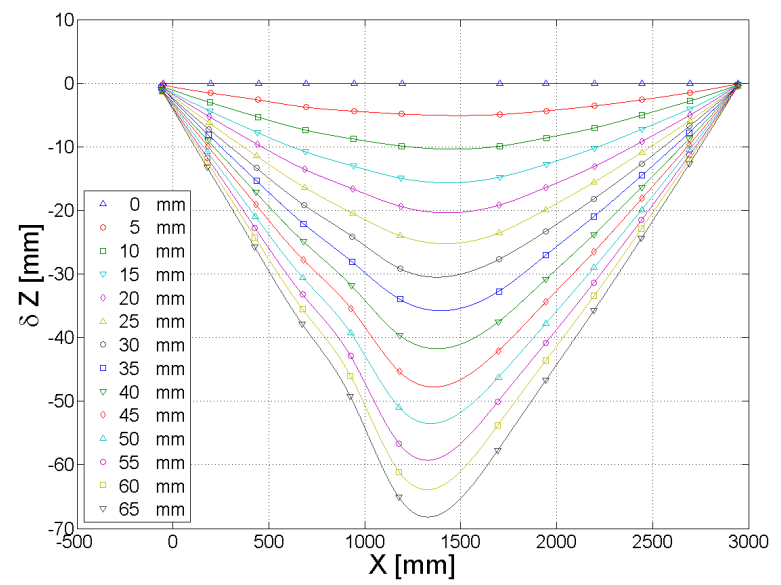

Figure 9. Cubic spline plots of the beam deflections for the first experiment using the photogrammetric reconstruction

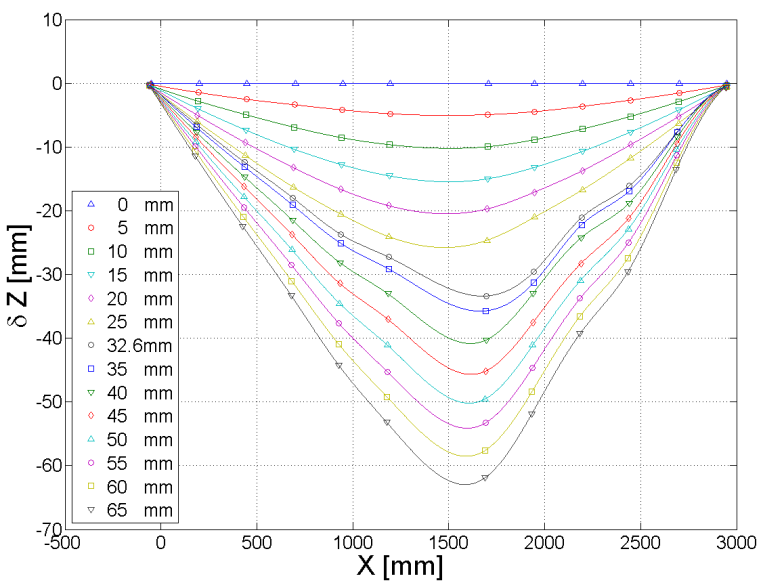

Figure 10. Cubic spline plots of the beam deflections for the second experiment using the photogrammetric reconstruction

The deflections computed from the photogrammetric reconstruction were also compared to the ones resulted from one of the laser scanners. From Table 2, it can be seen that the discrepancies between the two systems were similar for both experiments. The RMSE values of about $0.5 \mathrm{~mm}$ were acceptable, because they are comparable to the previously published precision of terrestrial laser scanning geometrically fitted data. From Figure 11 and Figure 12, it can also be seen that for the most part, the discrepancies in the estimated deflection values between the photogrammetric reconstruction and the laser scanner data was at plate \#7. This was the plate, which could not be observed by three consecutive cameras, and the cubic spline interpolation method did not correctly predict its deflections.

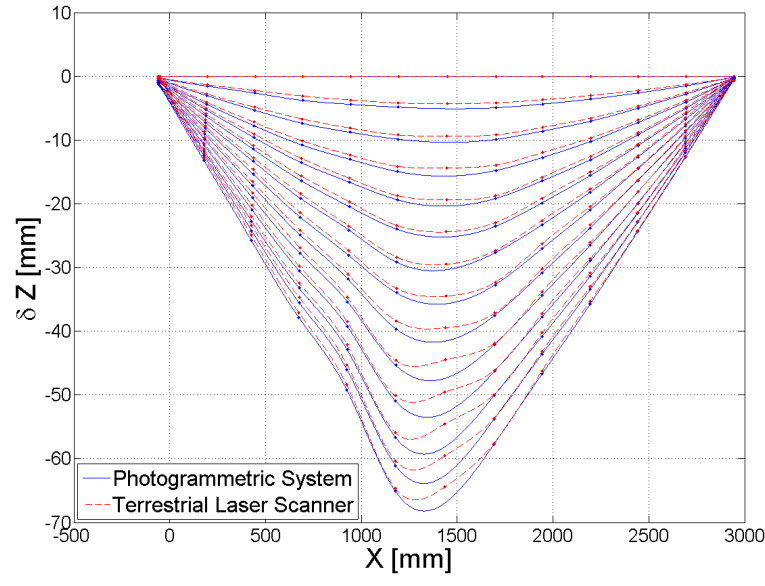

Figure 11. Cubic spline plots of the beam deflections computed using the photogrammetric reconstruction vs. the terrestrial laser scanner for the first experiment

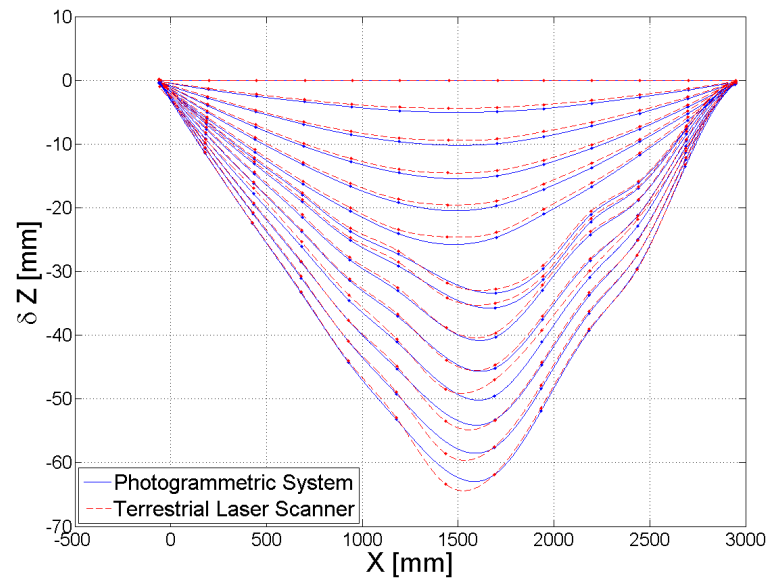

Figure 12. Cubic spline plots of the beam deflections computed using the photogrammetric reconstruction vs. the terrestrial laser scanner for the second experiment

\section{CONCLUSIONS AND RECOMMENDATIONS FOR FUTURE WORK}

This paper explored the use of consumer grade cameras and projectors for the deformation monitoring of structural elements. The aim of the two conducted experiments was to set up multiple digital cameras and projectors on a stable metal frame in order to be able to detect deflections in concrete beams caused by a hydraulic actuator. After performing a semiautomated photogrammetric reconstruction of the visible beam surfaces and of the full surfaces of almost all the metal plates, it was shown that sub-millimetre precision for the estimation of the beam deflections could be achieved in object space.

Future work will strive to achieve better results by both increasing the automation of the relative orientation estimation and by improving the precision of the reconstruction. These two goals could be fulfilled by using circular targets (for sub-pixel image coordinate measurement accuracy) with coded point numbers during the EOP estimation process. In addition, a high 
resolution (and potentially a high speed) camera will be added to the system in order to track the appearance and the enlargement of any cracks in the concrete through optical flow methods. Also, the cameras will be used in continuous shooting mode in order to detect deformations in concrete beams during dynamic load testing conditions.

\begin{tabular}{|c|c|c|c|c|}
\hline \multirow{2}{*}{} & \multicolumn{3}{|c|}{ Z Differences [mm] } \\
\cline { 2 - 5 } & \multicolumn{2}{|c|}{ Experiment 1 } & \multicolumn{2}{c|}{ Experiment 2 } \\
\cline { 2 - 5 } & Plate & Plate & Plate & Plate \\
& $\# 5$ & $\# 9$ & $\# 5$ & $\# 9$ \\
\hline Min & -0.24 & -0.25 & -1.77 & -0.00 \\
Max & 0.06 & 0.04 & 0.00 & 4.13 \\
Mean & -0.06 & -0.10 & -0.92 & 1.94 \\
Stdev & 0.11 & 0.08 & 0.56 & 1.66 \\
RMSE & 0.12 & 0.13 & 1.06 & 2.53 \\
\hline
\end{tabular}

Table 1. Statistical properties for the deflection differences between the photogrammetric system and the laser transducers for all observed epochs

\begin{tabular}{|c|c|c|}
\hline \multirow{2}{*}{} & \multicolumn{2}{|c|}{ Z Differences [mm] } \\
\cline { 2 - 3 } & Experiment 1 & Experiment 2 \\
\hline Min & -1.29 & -2.54 \\
Max & 0.88 & 3.19 \\
Mean & -0.22 & -0.21 \\
Stdev & 0.42 & 0.64 \\
RMSE & 0.48 & 0.67 \\
\hline
\end{tabular}

Table 2. Statistical properties for the deflection differences between the photogrammetric system and the laser scanner for all available plates and all observed epochs

\section{ACKNOWLEDGEMENTS}

The authors would like to thank the NSERC Strategic Grant Program and Alberta Innovates Technology Futures for funding this research project. These experiments would not have been possible without the assistance of the University of Calgary civil engineering staff, especially Dan Tilleman and Mirsad Berbic. Also, the help of Bruce Wright, Ting On Chan, Eunju Kwak, Zahra Lari and Mohannad Al-Durgham was irreplaceable.

\section{REFERENCES}

Besl, P.J. and McKay, N.D., 1992. A Method for Registration of 3-D Shapes. IEEE Transactions on Pattern Analysis and Machine Intelligence, 14(2): 239-256.

Bond, J., Chrzanowski, A. and Kim, D., 2008. Bringing GPS into harsh environments for fully automated deformation monitoring. GPS Solutions, 12(1): 1-11.

Chen, Y. and Medioni, G., 1992. Object modelling by registration of multiple range images. Image and Vision Computing, 10(3): 145-155.

Ebeling, A., Chow, J.C.K. and Teskey, W.F., 2011. Deformation Analysis of Terrestrial Monitoring Observations on Turtle Mountain, Alberta. Journal of Applied Geodesy, 5(1): 47-58.

Ebeling, A., Radovanovic, R. and Teskey, W.F., 2009. Deformation Analysis Using a Multi-Parameter Transformation. Geomatica, 63(3): 97-107.

Fraser, C.S. and Riedel, B., 2000. Monitoring the thermal deformation of steel beams via vision metrology. ISPRS Journal of Photogrammetry \& Remote Sensing, 55(2000): 268-276.

González-Auilera, D., Gómez-Lahoz, J. and Sánchez, J., 2008. A New Approach for Structural Monitoring of Large Dams with Three-Dimensional Laser Scanner. Sensors, 8(8): 5866-5883.

Gordon, S.J. and Lichti, D.D., 2007. Modeling Terrestrial Laser Scanner Data for Precise Structural Deformation Measurement. Journal of Surveying Engineering, 133(2): 72-80.

Gruen, A. and Akca, D., 2005. Least squares 3D surface and curve matching. ISPRS Journal of Photogrammetry \& Remote Sensing, 59(3): 151-174.

Inaudi, D., Vurpillot, S., Casanova, N. and Kronenberg, P., 1998. Structural monitoring by curvature analysis using interferometric fiber optic sensors. Smart Materials and Structures, 7(2): 199-208.

Jáuregui, D.V., White, K.R., Woodward, C.B. and Leitch, K.R., 2003. Noncontact Photogrammetric Measurement of Vertical Bridge Deflection. Journal of Bridge Engineering, 8(4): 212222.

Kim, D., Langley, R.B., Bond, J. and Chrzanowski, A., 2003. Local deformation monitoring using GPS in an open mine: initial study. GPS Solutions, 7(3): 176-185.

Lin, S.-Y., Mills, J.P. and Golsling, P.D., 2008 Videogrammetric Monitoring of As-Built Membrane Roof Structures. Photogrammetric Record, 23(122): 128-147.

Maas, H.-G. and Hampel, U., 2006. Photogrammetric Techniques in Civil Engineering Material Testing and Structure Monitoring. Photogrammetric Engineering \& Remote Sensing, 72(1): 39-45.

Mills, J.P., Newton, I. and Peirson, G.C., 2001. Pavement Deformation Monitoring in a Rolling Load Facility. Photogrammetric Record, 17(97): 7-24.

Monserrat, O. and Crosetto, M., 2008. Deformation measurement using terrestrial laser scanning data and least squares 3D surface matching. ISPRS Journal of Photogrammetry \& Remote Sensing, 63(2008): 142-154.

Park, H.S., Lee, H.M., Adeli, H. and Lee, I., 2007. A New Approach for Health Monitoring of Structures: Terrestrial Laser Scanning. Computer-Aided Civil and Infrastructure Engineering, 22(2007): 19-30.

Shen, S., Wu, Z., Yang, C., Wan, C., Tang, Y. and Wu, G., 2010. An Improved Conjugated Beam Method for Deformation Monitoring with Distributed Sensitive Fiber Optic Sensor. Structural Health Monitoring, 9(4): 361-378. 